\title{
Nedaplatin: a cisplatin derivative in cancer chemotherapy
}

\author{
This article was published in the following Dove Press journal: \\ Cancer Management and Research \\ 7 May 2013 \\ Number of times this article has been viewed
}

\section{Muneaki Shimada' \\ Hiroaki Itamochi' \\ Junzo Kigawa ${ }^{2}$}

'Department of Obstetrics and Gynecology, Tottori University School of Medicine, Yonago, Japan; ${ }^{2}$ Cancer Center, Tottori University Hospital, Yonago, Japan
Correspondence: Muneaki Shimada Department of Obstetrics and Gynecology, Tottori University School of Medicine, 36-I Nishimachi Yonago 683-8504, Japan

Tel +8I 859386647

Fax $+8 I 859386649$

Email mshimal2@med.tottori-u.ac.jp
Abstract: Nedaplatin, a cisplatin analog, has been developed to decrease the toxicities induced by cisplatin, such as nephrotoxicity and gastrointestinal toxicity. The dose of nedaplatin is determined by body surface area, not by the area under the curve (AUC). The recommended therapeutic dose is $80-100 \mathrm{mg} / \mathrm{m}^{2}$, although the pharmacokinetic profile of nedaplatin is similar to that of carboplatin. In our preliminary study, there was a favorable correlation between AUC and creatinine clearance (CL), suggesting that renal function should be considered when nedaplatin is administered. Ishibashi's formula, ie, Dose ${ }_{\mathrm{NDP}}=\mathrm{AUC} \times \mathrm{CL}_{\mathrm{NDP}}$ where $\mathrm{CL}_{\mathrm{NDP}}=0.0738 \times$ creatinine clearance +4.47 , would be predictable and useful for estimating the individual dose of nedaplatin. Several Phase II studies have suggested that nedaplatin might be a useful second analog, especially for patients with non-small cell lung cancer, esophageal cancer, uterine cervical cancer, head and neck cancer, or urothelial cancer. Further, nedaplatin was reported to be a useful chemotherapeutic agent with radiosensitizing properties; however, there is no Phase III study of nedaplatin, neither with chemotherapy nor with concurrent chemoradiotherapy, because nedaplatin is not commonly used throughout the world. Further evaluation in a randomized controlled trial is warranted to demonstrate definitively the activity of nedaplatin.

Keywords: nedaplatin, area under the curve, chemotherapy, concurrent chemoradiotherapy

\section{Introduction}

Although cisplatin is a potent anticancer agent, it often induces nephrotoxicity and gastrointestinal toxicity, which limits its clinical use. Nedaplatin (cis-diammineglycolatoplatinum), which is a second cisplatin analog, was developed in 1983 by Shionogi Pharmaceutical Company, Japan, to provide a treatment with effectiveness similar to that of cisplatin but with decreased renal and gastrointestinal toxicities. ${ }^{1,2}$ According to the National Comprehensive Cancer Network guidelines, cisplatin is categorized as having a high risk of emesis, whereas nedaplatin is reported to have a moderate emetic risk in the clinical practice guidelines of the Japan Society of Clinical Oncology.

Nedaplatin has the same ammine carrier ligands as cisplatin, but has a different leaving group, consisting of a five-membered ring structure in which glycolate is bound to the platinum ion as a bidentate ligand (Figure 1). Nedaplatin, which is a cisplatin analog with two ammine ligands, like carboplatin, is cross-resistant with cisplatin. ${ }^{3}$ Nedaplatin reacts with nucleosides to form a nucleoside-platinum complex, similar to cisplatin. It has been confirmed that the types of combined bases in nedaplatin after reaction with DNA are identical to those observed in cisplatin. After uptake 
<smiles>N[PH](N)(Cl)Cl</smiles><smiles>N[Pb]1(N)OCC(=O)O1</smiles>

Nedaplatin<smiles>N[PH]1(N)OC(=O)CCC1=O</smiles>

Carboplatin

Figure I Platinum agents (cisplatin, nedaplatin, and carboplatin).

into cells, the glycolate portion of nedaplatin is cleaved by hydrolysis, forming "active species 1" (Figure 2). Active species 1 interconverts between a series of other active species, all of which exist in equilibrium. Like cisplatin, the active species binds to DNA, thereby inhibiting DNA duplication. The plasma concentration profile of unbound platinum after a nedaplatin infusion has been reported to be similar to that of total platinum, and the protein binding of nedaplatin to be lower than that of cisplatin. ${ }^{4}$ Nedaplatin has a short elimination half-life (1.1-4.4 hours) and a pharmacokinetic profile similar to that of carboplatin. ${ }^{5}$

The dose-limiting toxicity of nedaplatin is myelosuppression, including leucopenia, anemia, and

primarily thrombocytopenia. ${ }^{6}$ In a Phase I study, two of five patients receiving nedaplatin at a dose of $120 \mathrm{mg} / \mathrm{m}^{2}$ developed grade 4 thrombocytopenia, in which nadir platelet counts were noted about three weeks after administration of nedaplatin, requiring 7-10 days for recovery. ${ }^{6}$ In contrast, leukopenia and anemia also occurred at higher doses of nedaplatin, but were milder than the thrombocytopenia. Based on the results of the Phase I study of single administration of nedaplatin, the maximum tolerated dose was established as $120 \mathrm{mg} / \mathrm{m}^{2}$. Consequently, nedaplatin was initially administered alone at doses of $100 \mathrm{mg} / \mathrm{m}^{2}$ intravenously every four weeks. The Phase I study of five days of continuous administration of nedaplatin showed that a dose of $75.5 \mathrm{mg} / \mathrm{m}^{2}$ would be feasible over a five-day period; ${ }^{7}$ however, it was concluded that continuous administration of nedaplatin would offer no advantage over single administration with a four-week interval, because the area under the curve (AUC) values for free platinum at $75.5 \mathrm{mg} / \mathrm{m}^{2}$ every five days and $100 \mathrm{mg} / \mathrm{m}^{2} /$ day were nearly equivalent.

Nedaplatin-induced nephrotoxicity was reported to be characterized by apoptosis and/or necrosis, with subsequent
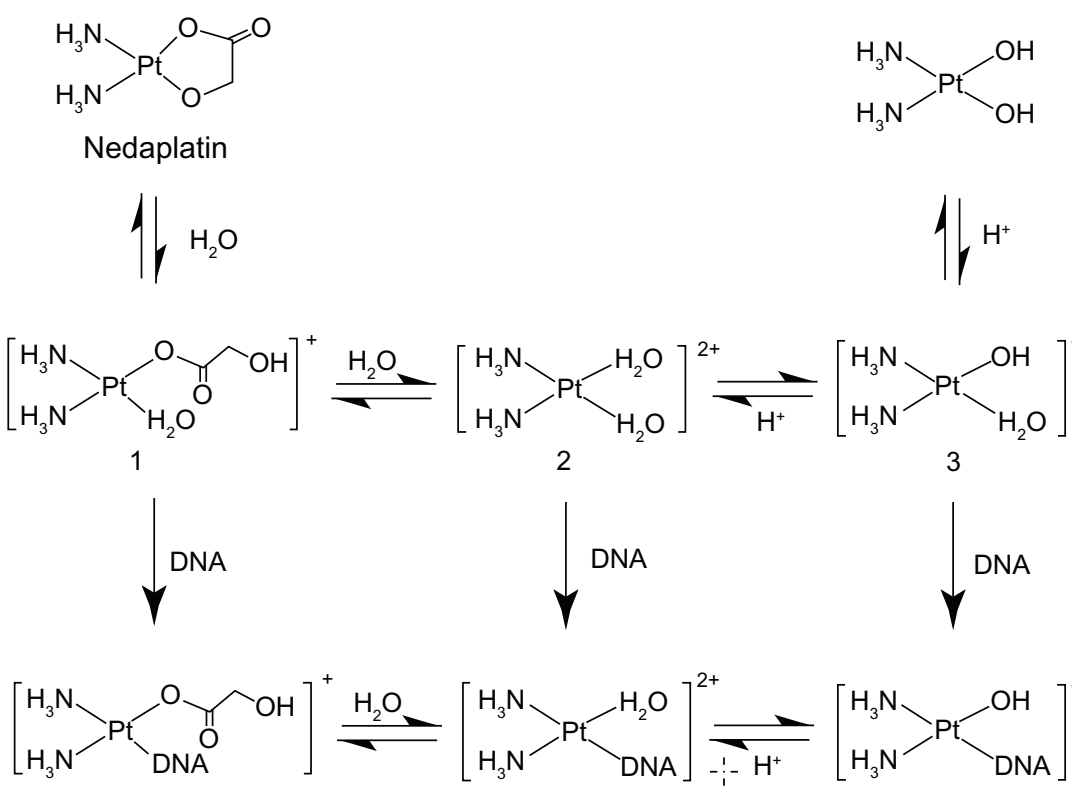

3
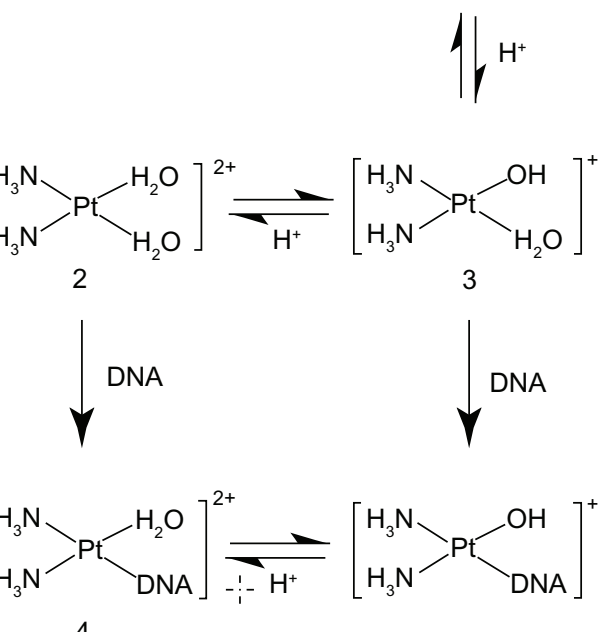

4

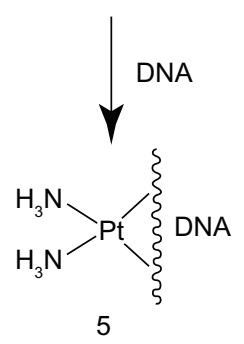

Figure 2 Postulated pathway for hydrolysis and DNA binding of nedaplatin 
regeneration and cystic dilatation, not only in the proximal tubules but also in the distal tubules and the collecting duct in a histopathological examination in rats. ${ }^{8}$ Further, deregulation of the genes encoding cytokeratins 14 and 19 reflects the characteristic renal papillary injury associated with nedaplatin. ${ }^{9}$ Nedaplatin may also cause nephrotoxicity at therapeutic doses, especially in patients with deteriorating renal function, while the abovementioned findings can lead to the establishment of an effective strategy for the safe use of nedaplatin in clinical practice.

\section{Optimal dosage based on renal function}

In anticancer chemotherapy, it is usual to use the maximum tolerated dose with respect to side effects, and serious side effects often occur, especially in patients exposed to high platinum concentrations. The optimal dosage of anticancer chemotherapeutic agents should be individualized by taking the pharmacokinetic variability into consideration. It is known that the AUC of platinum correlates with its anticancer efficacy and toxicity. ${ }^{10,11}$ The relationships between the AUC of platinum and efficacy and toxicity after carboplatin administration have been investigated. ${ }^{12}$ The formula for calculating the clearance of platinum has been reported for carboplatin, ${ }^{10,11}$ and the optimal dosing of carboplatin has been determined by the AUC using Calvert's formula in practice. Although the pharmacokinetic profile of nedaplatin is similar to that of carboplatin, administration of nedaplatin is determined by body surface area, not AUC. ${ }^{5}$

Ishibashi et al developed a simple formula based on renal function in adult Japanese cancer patients to predict the optimal dosage of nedaplatin. ${ }^{13}$ They measured unbound platinum concentrations in plasma after intravenous infusion of nedaplatin over 187 courses in 145 patients with lung, esophageal, uterine, cervical, or ovarian cancer undergoing clinical treatment. These data were divided into two sets, a model development data set of 94 courses and a validation data set of 93 courses. Regression analysis was applied to the relationship between the unbound platinum clearance of nedaplatin and the patients' renal function. Further, the predictability and usefulness of this formula were assessed by validation using the external data set of 93 courses obtained from 75 patients. They also obtained other unbound platinum concentrations in plasma after intravenous infusion of nedaplatin from 183 courses in 141 patients to clarify the pharmacokinetic profile of nedaplatin and to develop a population pharmacokinetic model. ${ }^{14}$ As a result, Ishibashi et al established a simple formula for predicting nedaplatin clearance based on pharmacokinetics:

$$
\begin{aligned}
\text { Dose }_{\mathrm{NDP}} & =\mathrm{AUC} \times \mathrm{CL}_{\mathrm{NDP}} \text {, where } \\
\mathrm{CL}_{\mathrm{NDP}} & =0.0738 \times \mathrm{CL}_{\mathrm{cr}}+4.47 \text { (Ishibashi's formula). }
\end{aligned}
$$

We evaluated this formula in 22 patients with uterine, cervical, or ovarian cancer, who underwent chemotherapy consisting of nedaplatin and irinotecan. ${ }^{14}$ Blood samples were collected at hours $0,1,2,4$ and 6 after the end of a $48-80 \mathrm{mg} / \mathrm{m}^{2}$ nedaplatin infusion, and free platinum concentrations were measured. The range of the observed AUC of nedaplatin was wide, and there were no relationships between the observed AUC and Dose ${ }_{\mathrm{NDP}}\left(\mathrm{mg} / \mathrm{m}^{2}\right)$ based on body surface area (Figure 3 ). In contrast, there was a favorable correlation between the observed AUC and the dose normalized by creatinine clearance (Figure 4). In addition, the predictive AUC correlated with the observed AUC (Figure 5). There was also a relationship between the observed AUC and the relative reduction ratio of platelets, although the dose of nedaplatin based on body surface area did not relate to the relative reduction ratio of platelets (Figure 6). We also confirmed that the observed AUC corresponded to the target AUC by dosing based on renal function, although there was no relationship between the observed AUC and Dose ${ }_{\mathrm{NDP}}\left(\mathrm{mg} / \mathrm{m}^{2}\right)$ based on body surface area. ${ }^{16}$ Consequently, our two previous clinical evaluations suggested that Ishibashi's formula would be predictable and useful for estimating the individual dose of nedaplatin.

\section{Clinical findings}

High anticancer activity has been reported for nedaplatin in Phase II studies of patients with non-small cell lung cancer (NSCLC), esophageal cancer, uterine cervical cancer, or head and neck cancer (Table 1).

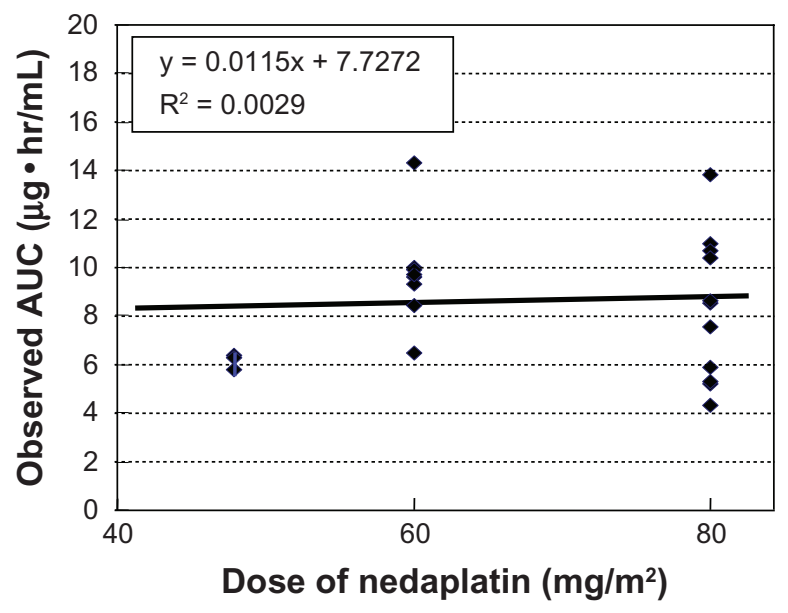

Figure 3 Observed AUC and dose of nedaplatin $\left(\mathrm{mg} / \mathrm{m}^{2}\right)$. Abbreviation: AUC, area under the curve. 


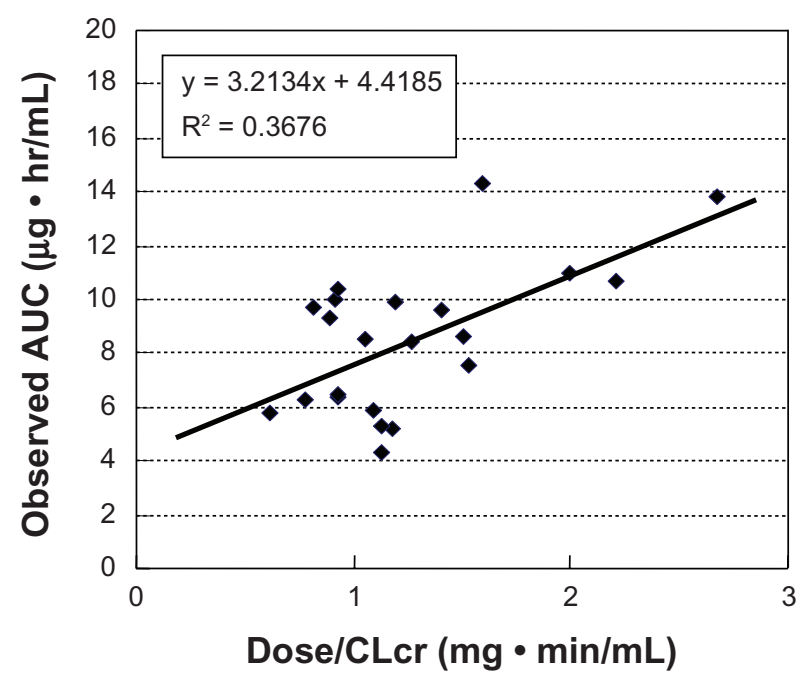

Figure 4 Observed AUC and clearance.

Note: There was favorable correlation between observed AUC and dose normalized by creatinine clearance.

Abbreviation: AUC, area under the curve.

\section{Non-small cell lung cancer}

At the time of diagnosis of NSCLC, the majority of patients have locally advanced or metastatic disease and a poor prognosis. These patients are recommended to undergo 4-6 cycles of platinum-based doublet chemotherapy as standard first-line treatment, with a response rate of about $30 \%$ and a median survival of $8-10$ months. ${ }^{17}$

In 44 patients with metastatic NSCLC, combination chemotherapy of docetaxel $60 \mathrm{mg} / \mathrm{m}^{2}$ and nedaplatin $80 \mathrm{mg} / \mathrm{m}^{2}$ showed a response rate of $50 \%$, which included 0 complete responses, 22 partial responses, 11 with stable

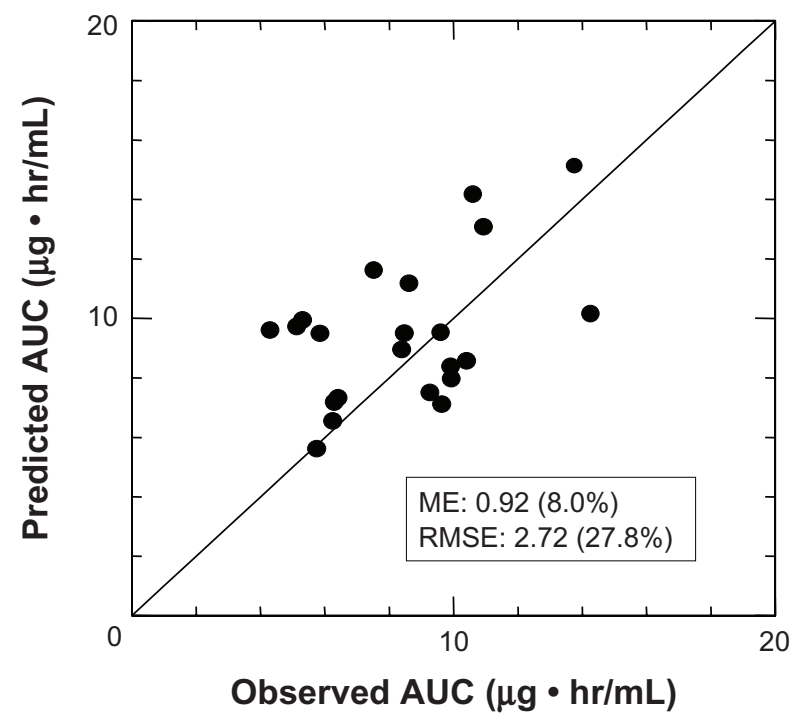

Figure 5 Predictive AUC and observed AUC.

Note: Predictive AUC was correlated with the observed AUC.

Abbreviations: AUC, area under the curve; ME, mean prediction error as a measure of bias; RMSE, root mean squared error as a measure of precision. disease, and 11 with progressive disease, with median progression-free survival and overall survival of 7.4 months and 13.0 months, respectively. ${ }^{18} \mathrm{~A}$ high response rate was achieved in patients with squamous cell carcinoma compared with that in those with adenocarcinoma (66.7\% versus $41.4 \%$ ). Forty-seven patients were treated with weekly combination chemotherapy of paclitaxel $90 \mathrm{mg} / \mathrm{m}^{2} /$ day on days 1,8 , and 15 and nedaplatin $80 \mathrm{mg} / \mathrm{m}^{2}$, and achieved a response rate of $53.2 \%$ and a median survival of 13 months. ${ }^{19}$ The incidence of grade $3 / 4$ hematologic toxicities was as follows: neutropenia (38.3\%), anemia (23.4\%), and thrombocytopenia (2.1\%). Combination chemotherapy including irinotecan $60 \mathrm{mg} / \mathrm{m}^{2}$ on days 1 and 8 and nedaplatin $100 \mathrm{mg} / \mathrm{m}^{2}$ every four weeks showed a response rate of $65.8 \%$ (25 with a partial response, nine with stable disease, and three with progressive disease) and a median survival of 13.9 months in 38 patients with unresectable NSCLC. ${ }^{20}$ All patients were aged 70 years or older in this Phase II study. Nineteen patients (50\%) had grade 4 neutropenia, and neutropenic fever occurred in 11 patients $(29 \%)$.

The same authors reported a Phase II study of combination chemotherapy consisting of nedaplatin $50 \mathrm{mg} / \mathrm{m}^{2}$ and irinotecan $60 \mathrm{mg} / \mathrm{m}^{2}$ on days 1 and 8 followed by $250 \mathrm{mg} /$ day of gefinitib, an epidermal growth factor receptor tyrosine kinase inhibitor, until tumor progression after completion of three cycles of nedaplatin-irinotecan chemotherapy in 28 elderly patients with unresectable NSCLC. ${ }^{21}$ The response rate for nedaplatin-irinotecan was $39.3 \%$, with one complete response, 10 patients with a partial response, 14 with stable disease, and three with progressive disease. Twenty-one patients received combination chemotherapy of nedaplatin and irinotecan followed by gefinitib, and had a response rate of $42.9 \%$ and a median survival of 8.7 months.

Sugiyama et al retrospectively assessed the efficacy and safety of gemcitabine $800 \mathrm{mg} / \mathrm{m}^{2}$ on days 1 and 8 and nedaplatin $80 \mathrm{mg} / \mathrm{m}^{2}$ on day 1 in 35 elderly patients (older than 75 years) with advanced NSCLC. ${ }^{22}$ The response rate was $45.7 \%$ and the median survival time was 14.0 months. Grade 3/4 hematologic toxicities were neutropenia (74.3\%), thrombocytopenia (48.6\%), and anemia (34.3\%).

Molecularly targeted agents are reported to play an important role in the treatment of patients with lung cancer. Bevacizumab in combination with paclitaxelcarboplatin chemotherapy offered significant prolongation of overall survival in patients with nonsquamous cell lung cancer. ${ }^{23}$ In patients with advanced NSCLC and high expression of epidermal growth factor receptor, 


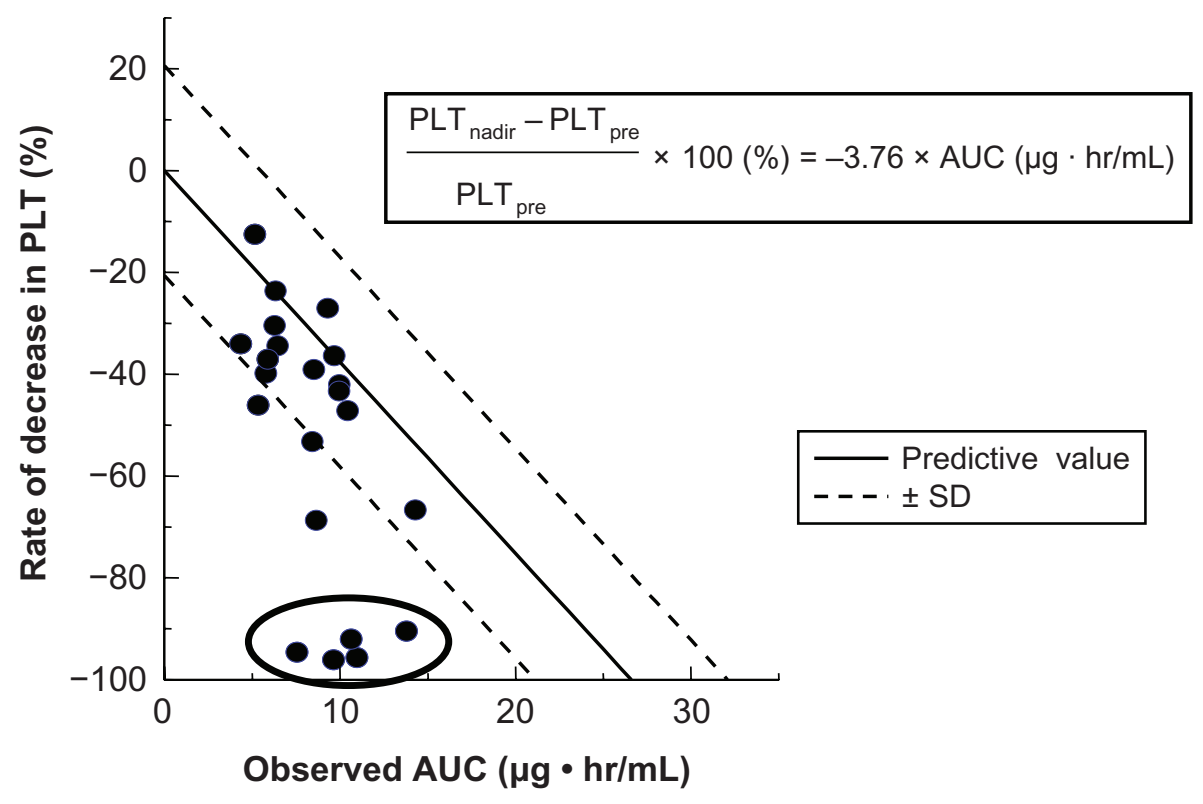

Figure 6 Observed AUC and relative reduction rate of platelet.

Notes: There was a relationship between the observed AUC and rate of decrease in platelets (PLT). Five patients, who were circled in figure, underwent concurrent chemoradiotherapy and showed unexpectedly grade 4 thrombocytopenia, and the predicted PLT did not follow the regression line.

Abbreviations: $\mathrm{PLT}_{\text {nadir }}$, the nadir of platelets count after nedaplatin administration; $\mathrm{PLT}_{\text {pre }}$, the platelets count before nedaplatin administration; $\mathrm{AUC}$, area under the curve; $\mathrm{SD}$, standard deviation.

cetuximab in combination with cisplatin-vinorelbine chemotherapy prolonged overall survival. ${ }^{24}$ Gefitinib and erlotinib have also demonstrated superiority in terms of progression-free-survival and tolerability in patients with NSCLC. ${ }^{25}$ Compared with placebo or best supportive care, maintenance chemotherapy with molecularly targeted agents is considered a promising strategy for patients with advanced NSCLC.

\section{Esophageal cancer}

Cisplatin with 5-fluorouracil is the most common standard chemotherapeutic regimen for advanced or metastatic

Table I Phase II studies of nedaplatin

\begin{tabular}{|c|c|c|c|c|}
\hline Author & Numbers & Line of chemotherapy & Regimen & Response rate \\
\hline \multicolumn{5}{|c|}{ Non-small cell lung cancer } \\
\hline Teramoto et $\mathrm{al}^{18}$ & 44 & First-line & DTX/NDP & $50.0 \%$ \\
\hline Hirose et al ${ }^{19}$ & 47 & First-line & Weekly PTX/NDP & $53.2 \%$ \\
\hline Oshita et $\mathrm{al}^{20}$ & 38 & First-line & CPT-II/NDP & $65.8 \%$ \\
\hline Oshita et $\mathrm{al}^{21}$ & 28 & First-line & CPT-I I/NDP then gefinitib & $42.9 \%$ \\
\hline \multicolumn{5}{|l|}{ Esophageal cancer } \\
\hline Cao et $\mathrm{al}^{30}$ & 46 & First-line & PTX/NDP & $41.7 \%$ \\
\hline Gong et $\mathrm{a}^{31}$ & 49 & First-line & PTX/NDP & $43.6 \%$ \\
\hline Guo et $\mathrm{al}^{32}$ & 43 & First-line & DTX/NDP/5-FU & $62.8 \%$ \\
\hline Jingu et $\mathrm{a}^{34}$ & 30 & First-line & CCRT with NDP/5-FU & $73.3 \%$ \\
\hline \multicolumn{5}{|c|}{ Uterine cervical cancer } \\
\hline Takekuma et a $\mathrm{a}^{139}$ & 50 & First/second-line & PTX/NDP & $44.4 \%$ \\
\hline Yamaguchi et $\mathrm{al}^{40}$ & 68 & First-line & CPT-I I/NDP & $75.8 \%$ \\
\hline Tsuda et $\mathrm{al}^{41}$ & 27 & First-line & CPT-II/NDP & $59.0 \%$ \\
\hline Yokoyama et a ${ }^{14}$ & 45 & First-line & CCRT with weekly NDP & $88.9 \%$ \\
\hline \multicolumn{5}{|l|}{ Head and neck cancer } \\
\hline Ohashi et a ${ }^{49}$ & 31 & First-line & CCRT with S-I/NDP & $81.0 \%$ \\
\hline Kurita et al ${ }^{56}$ & 30 & First-line & DTX/NDP & $33.3 \%$ \\
\hline \multicolumn{5}{|l|}{ Urothelial cancer } \\
\hline Kitamura et $a^{55}$ & 45 & Second-line & PTX/IFM/NDP & $40.0 \%$ \\
\hline Shinohara et a $\left.\right|^{53}$ & 32 & Second-line & PTX/IFM/NDP & $75.0 \%$ \\
\hline
\end{tabular}

Abbreviations: DTX, docetaxel hydrate; NDP, nedaplatin; PTX, paclitaxel; CPT-II, irinotecan hydrochloride hydrate; 5-FU, fluorouracil; CCRT, concurrent chemoradiotherapy; S-I, tegafur, gimeracil, oteracil potassium; IFM, ifosfamide. 
esophageal cancer. This standard regimen induces a response rate of $33 \%-35 \%$ and a median survival of $6-8$ months, but a complete response is rare. ${ }^{26}$ Further, this chemotherapeutic regimen is inconvenient because of the need for continuous administration over 5 days, and it sometimes causes renal dysfunction. To reduce the renal and gastrointestinal toxicity found with cisplatin, nedaplatin is used in Japan to treat patients with advanced or recurrent esophageal cancer. Paclitaxel and docetaxel have shown overall response rates as single agents of $32 \%$ and $17 \%-20 \%$ in the treatment of esophageal cancer. ${ }^{27-29}$ Combination chemotherapy including paclitaxel $175 \mathrm{mg} / \mathrm{m}^{2}$ and nedaplatin $80 \mathrm{mg} / \mathrm{m}^{2}$ had an overall response rate of $41.7 \%$, and median time to progression and overall survival of 6.1 months and 11.5 months, respectively, in 46 patients with advanced esophageal cancer. ${ }^{30}$ Six patients (13.0\%) had grade $3 / 4$ anemia, nine $(19.6 \%)$ had grade 3 neutropenia, and two $(4.3 \%)$ had grade 3 thrombocytopenia. In the other Phase II study of combination chemotherapy comprising paclitaxel $175 \mathrm{mg} / \mathrm{m}^{2}$ and nedaplatin $80 \mathrm{mg} / \mathrm{m}^{2}$ in 39 patients with metastatic esophageal cancer, the overall response rate, time to progression, and overall survival were 43.6\%, 6.1 months, and 10.3 months, respectively. ${ }^{31}$ Triple combination chemotherapy, including docetaxel $80 \mathrm{mg} / \mathrm{m}^{2}$, nedaplatin $100 \mathrm{mg} / \mathrm{m}^{2}$, and 5-fluorouracil $375 \mathrm{mg} / \mathrm{m}^{2}$ as a bolus followed by $2600 \mathrm{mg} / \mathrm{m}^{2}$ via 46-hour infusion, had a response rate of $62.8 \%$, with two complete responses, 25 partial responses, nine cases of stable disease, and seven cases of progressive disease in 43 patients with advanced esophageal cancer. ${ }^{32}$ The incidence of grade $3 / 4$ neutropenia was $20.9 \%$, with $4.7 \%$ for grade $3 / 4$ febrile neutropenia and $7.0 \%$ for grade $3 / 4$ thrombocytopenia. Fujita et al retrospectively assessed the efficacy and safety of biweekly combination chemotherapy consisting of docetaxel $30 \mathrm{mg} / \mathrm{m}^{2}$ and nedaplatin $40 \mathrm{mg} / \mathrm{m}^{2}$ for 11 patients with recurrent esophageal cancer compared with combination chemotherapy consisting of 5-fluorouracil $400 \mathrm{mg} / \mathrm{m}^{2}$ on days $1-5$ and cisplatin $40 \mathrm{mg} / \mathrm{m}^{2}$ every four weeks for 10 patients. ${ }^{33} \mathrm{Com}-$ bination chemotherapy of docetaxel and nedaplatin showed a response rate of $36.3 \%$ and median overall survival of 234 days with a median follow-up of 234 days, with a response rate of $10.0 \%$ and median overall survival of 378 days, with a median follow-up of 279 days, in the group receiving 5-fluorouracil and cisplatin. There was no significant difference between the two groups. Jingu et al reported on the efficacy and safety of radiotherapy (60 Gy/30 fractions over six weeks) combined with two cycles of combination chemotherapy comprising 5 -fluorouracil $500 \mathrm{mg} / \mathrm{m}^{2}$ per 24 hours for five days and nedaplatin $70 \mathrm{mg} / \mathrm{m}^{2}$ on day 1 every 3 weeks for 30 patients with postoperative locoregional recurrence of esophageal cancer. ${ }^{34}$ The rate of completion of this treatment without reduction of chemotherapy was $76.7 \%$. The five-year survival rate was $27.0 \%$, with a median survival duration of 21.0 months. Major toxicities in the acute phase were grade 3 neutropenia $(30.0 \%)$, grade 4 thrombocytopenia $(3.3 \%)$, grade $3 / 4$ diarrhea $(6.6 \%)$, and grade 3 heartburn or mucositis (3.3\%). Only one patient developed major late toxicity and died six months after completion of the protocol-scheduled treatment because of serious pericardial effusion.

\section{Uterine cervical cancer}

The randomized Gynecologic Oncology Group Phase III study (protocol 209) revealed that combination chemotherapy of paclitaxel and cisplatin was the optimal cisplatin doublet regimen for patients with advanced or recurrent uterine cervical cancer. ${ }^{35}$ However, because advanced or recurrent uterine cervical cancer sometimes causes renal dysfunction, chemotherapy containing cisplatin is limited due to the cisplatin-induced nephrotoxicity seen in patients with these diseases. Nedaplatin demonstrated the same antitumor activity as cisplatin in an ex vivo study of uterine cervical cancer. ${ }^{36,37}$ In Japan, nedaplatin is commonly used for patients with advanced or recurrent uterine cervical cancer. The response rate for single chemotherapy using nedaplatin was $46.3 \%$ for cervical cancer. ${ }^{38}$ Takekuma et al reported that combination chemotherapy of paclitaxel $175 \mathrm{mg} / \mathrm{m}^{2}$ and nedaplatin $80 \mathrm{mg} / \mathrm{m}^{2}$ every four weeks had a response rate of $44.4 \%$ (11 complete responses and eight partial responses), a median progression-free-survival of 7.5 months, and a median overall survival of 15.7 months in 45 patients with advanced or recurrent uterine cervical cancer. ${ }^{39}$ The incidence of grade $3 / 4$ hematologic toxicity was $16.7 \%$ for neutropenia, $18.4 \%$ for anemia, and $2.0 \%$ for febrile neutropenia, but there was no grade 3/4 thrombocytopenia. The Japanese Gynecologic Oncology Group evaluated the efficacy and safety of combination chemotherapy comprising irinotecan $60 \mathrm{mg} / \mathrm{m}^{2}$ on days 1 and 9 and nedaplatin $80 \mathrm{mg} / \mathrm{m}^{2}$ every three weeks as neoadjuvant chemotherapy for patients with stage IB2-IIB uterine cervical squamous cell carcinoma. ${ }^{40}$ The response rate was $75.8 \%$, which included two patients with a complete response, 48 with a partial response, 12 with stable disease, and 0 with progressive disease, with four patients not evaluated. The incidence of grade $3 / 4$ hematologic toxicity was $72.2 \%$ for neutropenia, $13.6 \%$ for anemia, and $7.6 \%$ for thrombocytopenia. The most common grade $3 / 4$ nonhematologic toxicity was diarrhea (6.1\%). Another Phase I/II study of combination chemotherapy using 
irinotecan $50 \mathrm{mg} / \mathrm{m}^{2}$ on days 1 and 8 and nedaplatin $80 \mathrm{mg} / \mathrm{m}^{2}$ every four weeks showed a response rate of $59 \%$, median progression-free-survival of 161 days, and median overall survival of 415 days in 27 patients with advanced or recurrent uterine or cervical cancer. ${ }^{41}$

Concurrent chemoradiotherapy with weekly cisplatin $40 \mathrm{mg} / \mathrm{m}^{2}$ is accepted as the standard treatment for locally advanced uterine cervical cancer. Several in vitro and in vivo studies have reported that nedaplatin in combination with radiotherapy was highly effective for uterine cervical cancer, ${ }^{42,43}$ suggesting the radiosensitizing properties of nedaplatin. Because nedaplatin does not require hydration and has minimal nephrotoxicity and gastrointestinal toxicity, concurrent chemoradiotherapy with nedaplatin may be a more convenient treatment for patients with locally advanced uterine cervical cancer compared with cisplatin-based concurrent chemoradiotherapy. Yokoyama et al reported the efficacy and safety of chemoradiotherapy concurrent with nedaplatin $30 \mathrm{mg} / \mathrm{m}^{2} /$ week in a Phase II study of 45 patients with uterine cervical cancer. ${ }^{44}$ Forty patients $(88.9 \%)$ achieved an objective response, 36 patients achieved a complete response, and four achieved a partial response. With a median follow-up duration of 29 months, the three-year progression-free survival rate and overall survival rate were $58.7 \%$ and $78.0 \%$, respectively. Three patients $(6.7 \%)$ had grade 4 neutropenia, two $(4.4 \%)$ had grade 3 diarrhea, and one $(2.2 \%)$ had grade 3 nausea/vomiting.

\section{Head and neck cancer}

Given that preservation of organ function is important for maintaining quality of life, concurrent chemoradiotherapy has been frequently used in patients with head and neck cancer. A meta-analysis including 17,346 patients demonstrated a $6.5 \%$ absolute survival benefit for concurrent chemoradiotherapy and an 11\% survival benefit for chemoradiotherapy concurrent with cisplatin $100 \mathrm{mg} / \mathrm{m}^{2}{ }^{25}$ Although the additional benefit of 5-fluorouracil when added to chemoradiotherapy concurrent with cisplatin was limited, ${ }^{45,46}$ chemoradiotherapy concurrent with cisplatin and 5-fluorouracil is one of the most common treatments for patients with head and neck cancer. To limit the toxicities associated with cisplatin and 5-fluorouracil, several agents, such as nedaplatin and $\mathrm{S}-1$, can be used to replace cisplatin or 5-fluorouracil in Japan.

S-1 is a novel oral anticancer agent, consisting of tegafur, 5-chloro-2, 4-dehydroxyprimidine (gimeracil), and potassium oxonate (oteracil) at a 1:0.4:1 molar concentration ratio. Tegafur is hydroxylated and converted to 5-fluorouracil by microsomal enzymes in the liver. Gimeracil is a competitive inhibitor of dihydropyrimidine dehydrogenase that prevents rapid degradation of 5-fluorouracil and maintains effective 5-fluorouracil levels in plasma and tumor tissue. Gimeracil also has a radiosensitizing effect by inhibiting the repair of double-stranded DNA breaks. ${ }^{47}$ Oteracil is a competitive inhibitor of orotate phosphoribosyltransferase, which inhibits phosphorylation of 5-fluorouracil in the gastrointestinal tract and reduces the serious gastrointestinal toxicity associated with 5-fluorouracil. ${ }^{48}$ Consequently, S-1 may potentiate the antitumor activity of 5-fluorouracil and decrease the toxicities associated with 5-fluorouracil. Moreover, S-1 chemotherapy is more convenient than continuous intravenous administration of 5-fluorouracil. Ohashi et al evaluated the efficacy and safety of chemoradiotherapy concurrent with S-1 $80 \mathrm{mg} / \mathrm{m}^{2}$ orally for two weeks and nedaplatin $90-100 \mathrm{mg} / \mathrm{m}^{2}$ on day 4 in 31 patients with head and neck cancer. ${ }^{49}$ Two cycles of concomitant chemotherapy with S-1 and nedaplatin were scheduled in this study. Although all patients received one cycle of concomitant chemotherapy, only 15 patients received the protocol-scheduled two cycles of chemotherapy. Chemoradiotherapy concurrent with S-1 and nedaplatin achieved an overall complete response of $81 \%$ and a two-year overall survival of $96 \%$. Six patients $(19.4 \%$ ) had grade $3 / 4$ neutropenia, one (3.2\%) had grade 3 anemia, and one (3.2\%) had grade 3 thrombocytopenia. No patients had grade 4 nonhematological toxicity. Eight patients $(25.8 \%)$ had grade 3 dermatitis, six (19.4\%) had grade 3 mucositis, and one (3.2\%) had grade 3 nausea/vomiting.

\section{Urothelial cancer}

Cisplatin-based combination chemotherapy, such as methotrexate, vinblastine, doxorubicin, and cisplatin (MVAC) or gemcitabine-cisplatin, is the accepted standard first-line chemotherapy for patients with metastatic urothelial cancer. Gemcitabine-cisplatin chemotherapy showed the same response rate as that achieved with MVAC chemotherapy (approximately 40\%-50\%), but with less toxicity. ${ }^{50,51}$

In contrast, there is no standard chemotherapeutic regimen for pretreated patients with metastatic urothelial cancer. Triple cisplatin-based combination chemotherapy, including paclitaxel, ifosfamide, and gemcitabine, may provide higher response rates and longer survival than can doublet combination chemotherapy. ${ }^{52,53}$ Using the histoculture drug response assay, nedaplatin had a higher inhibition index than cisplatin in all tissue specimens from 12 patients with urothelial cancer, ${ }^{54}$ suggesting that nedaplatin can be effective for patients refractory to cisplatin-based chemotherapy, 
although nedaplatin is cross-resistant to cisplatin. Triple combination chemotherapy of paclitaxel $175 \mathrm{mg} / \mathrm{m}^{2}$, ifosfamide $1500 \mathrm{mg} / \mathrm{m}^{2}$ on days $1-3$, and nedaplatin $70 \mathrm{mg} / \mathrm{m}^{2}$ showed a response rate of $75.0 \%$, median progression-free-survival of eight months, and median overall survival of 22 months in 32 patients with metastatic urothelial cancer. Eight patients (25\%) had grade $3 / 4$ thrombocytopenia and six patients (19\%) had grade $3 / 4$ anemia, but all patients had grade 3/4 neutropenia. Additionally, all patients experienced grade 3 alopecia. Another Phase II study of paclitaxel $175 \mathrm{mg} / \mathrm{m}^{2}$ and ifosfamide $1500 \mathrm{mg} / \mathrm{m}^{2}$ on days $1-3$ and nedaplatin $70 \mathrm{mg} / \mathrm{m}^{2}$ was reported to have a response rate of $40.0 \%$, median progression-free survival of 4.0 months, and median overall survival of 8.9 months in 45 patients with metastatic urothelial cancer. ${ }^{55}$ This Phase II study included 10 patients (22.2\%) who had previously received gemcitabine-cisplatin chemotherapy. Forty-three patients $(95.6 \%)$ experienced grade $3 / 4$ neutropenia, while grade $3 / 4$ thrombocytopenia and anemia were seen in eight (17.8\%) and seven (15.6\%) patients, respectively.

\section{Conclusion}

Nedaplatin may be a useful second cisplatin analog for decreasing cisplatin-induced toxicities, especially in patients with NSCLC, esophageal cancer, uterine cervical cancer, head and neck cancer, or urothelial cancer. The dose of nedaplatin is determined from body surface area, not the AUC, and the recommended therapeutic dose is $80-100 \mathrm{mg} / \mathrm{m}^{2}$, although the pharmacokinetic profile of nedaplatin is similar to that of carboplatin. Consequently, renal function should be considered when nedaplatin is administered, in order to reduce severe nedaplatin-induced toxicities as far as possible. Unfortunately, since this second cisplatin analog is not commonly used, and there is no Phase III study of nedaplatin including chemoradiotherapy. To demonstrate the activity of nedaplatin definitively, further evaluation in a randomized controlled trial is warranted.

\section{Disclosure}

The authors report no conflicts of interest in this work.

\section{References}

1. Kameyama Y, Okazaki N, Nakagawa M, Koshida H, Nakamura M, Gemba M. Nephrotoxicity of a new platinum compound, 254-S, evaluated with rat kidney cortical slices. Toxicol Lett. 1990;52(1):15-24.

2. Niioka T, Uno T, Yasui-Furukori N, et al. Pharmacokinetics of lowdose nedaplatin and validation of AUC prediction in patients with non-small-cell lung carcinoma. Cancer Chemother Pharmacol. 2007; 59(5):575-580.
3. Lebwohl D, Canetta R. Clinical development of platinum complexes in cancer therapy: an historical perspective and an update. Eur J Cancer. 1998;34(10):1522-1534.

4. Ota K, Oguma T, Shimamura K. Pharmacokinetics of platinum in cancer patients following intravenous infusion of cis-diammine(glycolato) platinum, 254-S. Anticancer Res. 1994;14(3B):1383-1387.

5. SasakiY, Tamura T, Eguchi K, et al. Pharmacokinetics of (glycolate- $0,0^{\prime}$ )diammine platinum (II), a new platinum derivative, in comparison with cisplatin and carboplatin. Cancer Chemother Pharmacol. 1989;23(4):243-246.

6. Ota $\mathrm{K}$, Wakui A, Majima H, et al. Phase I study of a new platinum complex 254-S, cis-diammine (glycolato)-platinum (II). Gan To Kagaku Ryoho. 1992;19(6):855-861. Japanese.

7. Sasaki Y, Amano T, Morita M, et al. Phase I study and pharmacological analysis of cis-diammine(glycolato)platinum (254-S; NSC 375101D) administered by 5 -day continuous intravenous infusion. Cancer Res. 1991;51(5):1472-1477.

8. Uehara T, Yamate J, Torii M, Maruyama T. Comparative nephrotoxicity of cisplatin and nedaplatin: mechanisms and histopathological characteristics. J Toxicol Pathol. 2011;24(2):87-94.

9. Lieberthal W, Triaca V, Levine J. Mechanisms of death induced by cisplatin in proximal tubular epithelial cells: apoptosis versus necrosis. Am J Physiol. 1996;270(4):F700-F708.

10. Calvert AH, Newell DR, Gumbrell LA, et al. Carboplatin dosage: prospective evaluation of a simple formula based on renal function. J Clin Oncol. 1989;7(11):1748-1756.

11. Chatelut E, Canal P, Brunner V, et al. Prediction of carboplatin clearance from standard morphological and biological patient characteristics. J Natl Cancer Inst. 1995;87(8):573-580.

12. van Warmerdam LJ, Rodenhuis S, ten Bokkel Huinink WW, Maes RA, Beijnen JH. The use of the Calvert formula to determine the optimal carboplatin dosage. J Cancer Res Clin Oncol. 1995;121(8): 478-486.

13. Ishibashi T, Yano Y, Oguma T. A formula for predicting optimal dosage of nedaplatin based on renal function in adult cancer patients. Cancer Chemother Pharmacol. 2002;50(3):230-236.

14. Ishibashi T, Yano Y, Oguma T. Population pharmacokinetics of platinum after nedaplatin administration and model validation in adult patients. Br J Clin Pharmacol. 2003;56(2):205-213.

15. Sato S, Fujiwara H, Oishi T, et al. Evaluation of a formula for individual dosage of nedaplatin based on renal function. Cancer Chemother Pharmacol. 2012;69(3):599-603.

16. Shimada M, Fujiwara H, Sato S, et al. Area under the curve calculation of nedaplatin dose used in combination chemotherapy with irinotecan in a phase I study of gynecologic malignancies. Cancer Chemother Pharmacol. 2012;70(1):33-38.

17. Schiller JH, Harrington D, Belani CP, et al. Comparison of four chemotherapy regimens for advanced non-small-cell lung cancer. N Engl J Med. 2002;346(2):92-98.

18. Teramoto K, Asada Y, Ozaki Y, et al. A phase II study of docetaxel plus nedaplatin in patients with metastatic non-small-cell lung cancer. Cancer Chemother Pharmacol. 2012;70(4):531-537.

19. Hirose T, Sugiyama T, Kusumoto S, et al. Phase II study of the combination of nedaplatin and weekly paclitaxel in patients with advanced non-small cell lung cancer. Anticancer Res. 2009;29(5):1733-1738.

20. Oshita F, Yamada K, Saito H, Noda K, Hamanaka N, Ikehara M. Phase II study of nedaplatin and irinotecan for elderly patients with advanced non-small cell lung cancer. J Exp Ther Oncol. 2004;4(4):343-348.

21. Oshita F, Yamada K, Saito H, Noda K. Phase II study of nedaplatin and irinotecan followed by gefitinib for elderly patients with unresectable non-small cell lung cancer. Cancer Chemother Pharmacol. 2008;62(3): 465-470.

22. Sugiyama T, Hirose T, Nakashima M, et al. Evaluation of the efficacy and safety of the combination of gemcitabine and nedaplatin for elderly patients with advanced non-small-cell lung cancer. Oncology. 2011;81(3-4):273-280. 
23. Sandler A, Gray R, Perry MC, et al. Paclitaxel-carboplatin alone or with bevacizumab for non-small-cell lung cancer. N Engl J Med. 2006; 355(24):2542-2550.

24. Pirker R, Pereira JR, von Pawel J, et al. EGFR expression as a predictor of survival for first-line chemotherapy plus cetuximab in patients with advanced non-small-cell lung cancer: analysis of data from the phase 3 FLEX study. Lancet Oncol. 2012;13(1):33-42.

25. Rosell R, Carcereny E, Gervais R, et al. Erlotinib versus standard chemotherapy as first-line treatment for European patients with advanced EGFR mutation-positive non-small-cell lung cancer (EURTAC): a multicentre, open-label, randomised phase 3 trial. Lancet Oncol. 2012;13(3):239-246.

26. Ilson DH. Oesophageal cancer: new developments in systemic therapy. Cancer Treat Rev. 2003;29(6):525-532.

27. Ajani JA, Ilson DH, Daugherty K, Pazdur R, Lynch PM, Kelsen DP. Activity of Taxol in patients with squamous cell carcinoma and adenocarcinoma of the esophagus. J Natl Cancer Inst. 1994;86(14): 1086-1091.

28. Heath EI, Urba S, Marshall J, Piantadosi S, Forastiere AA. Phase II trial of docetaxel chemotherapy in patients with incurable adenocarcinoma of the esophagus. Invest New Drugs. 2002;20(1):95-99.

29. Muro K, Hamaguchi T, Ohtsu A, et al. A phase II study of single-agent docetaxel in patients with metastatic esophageal cancer. Ann Oncol. 2004;15(6):955-959.

30. Cao W, Xu C, Lou G, et al. A phase II study of paclitaxel and nedaplatin as first-line chemotherapy in patients with advanced esophageal cancer. Jpn J Clin Oncol. 2009;39(9):582-587.

31. Gong Y, Ren L, Zhou L, et al. Phase II evaluation of nedaplatin and paclitaxel in patients with metastatic esophageal carcinoma. Cancer Chemother Pharmacol. 2009;64(2):327-333.

32. Guo JF, Zhang B, Wu F, et al. A phase II trial of docetaxel plus nedaplatin and 5-fluorouracil in treating advanced esophageal carcinoma. Chin J Cancer. 2010;29(3):321-324.

33. Fujita Y, Hiramatsu M, Kawai M, Sumiyoshi K, Nishimura H, Tanigawa N. Evaluation of combined docetaxel and nedaplatin chemotherapy for recurrent esophageal cancer compared with conventional chemotherapy using cisplatin and 5-fluorouracil: a retrospective study. Dis Esophagus. 2008;21(6):496-501

34. Jingu K, Matsushita H, Takeda K, et al. Long-term results of radiotherapy combined with nedaplatin and 5-fluorouracil for postoperative locoregional recurrent esophageal cancer: update on a phase II study. BMC Cancer. 2012;12:542.

35. Monk BJ, Sill MW, McMeekin DS, et al. Phase III trial of four cisplatincontaining doublet combinations in stage IVB, recurrent, or persistent cervical carcinoma: a Gynecologic Oncology Group study. J Clin Oncol. 2009;27(28):4649-4655.

36. Monk BJ, Alberts DS, Burger RA, et al. In vitro phase II comparison of the cytotoxicity of a novel platinum analog, nedaplatin (254-S), with that of cisplatin and carboplatin against fresh, human cervical cancers. Gynecol Oncol. 1998;71(2):308-312.

37. Sasaki Y, Shinkai T, Eguchi K, et al. Prediction of the antitumor activity of new platinum analogs based on their ex vivo pharmacodynamics as determined by bioassay. Cancer Chemother Pharmacol. 1991;27(4): 263-270.

38. Kato T, Nishimura H, Yakushiji M, et al. Phase II study of 254-S (cis-diammine glycolato platinum) for gynecological cancer. Gan To Kagaku Ryoho. 1992;19:695-701. Japanese.

39. Takekuma M, Hirashima Y, Ito K, et al. Phase II trial of paclitaxel and nedaplatin in patients with advanced/recurrent uterine cervical cancer: a Kansai Clinical Oncology Group study. Gynecol Oncol. 2012;126(3): 341-345.

40. Yamaguchi S, Nishimura R, Yaegashi N, et al. Phase II study of neoadjuvant chemotherapy with irinotecan hydrochloride and nedaplatin followed by radical hysterectomy for bulky stage Ib2 to IIb, cervical squamous cell carcinoma: Japanese Gynecologic Oncology Group study (JGOG 1065). Oncol Rep. 2012;28(2):487-493.
41. Tsuda H, Hashiguchi Y, Nishimura S, et al. Phase I-II study of irinotecan (CPT-11) plus nedaplatin (254-S) with recombinant human granulocyte colony-stimulating factor support in patients with advanced or recurrent cervical cancer. Br J Cancer. 2004;91(6):1032-1037.

42. Nakamura Y, Hasegawa M, Hayakawa K, et al. Induction of p53dependent apoptosis in vivo by nedaplatin and ionizing radiation. Oncol Rep. 2000;7(2):261-265.

43. Tanaka T, Yukawa K, Umesaki N. Radiation reduces carboplatin sensitivity and enhances nedaplatin sensitivity in cervical squamous cell carcinoma in vitro. Eur J Gynaecol Oncol. 2007;28(5): 352-355.

44. Yokoyama Y, Takano T, Nakahara K, et al. A phase II multicenter trial of concurrent chemoradiotherapy with weekly nedaplatin in advanced uterine cervical carcinoma: Tohoku Gynecologic Cancer Unit Study. Oncol Rep. 2008;19(6):1551-1556.

45. Pignon JP, le Maître A, Maillard E, Bourhis J; MACH-NC Collaborative Group. Meta-analysis of chemotherapy in head and neck cancer (MACH-NC): an update on 93 randomised trials and 17,346 patients. Radiother Oncol. 2009;92(1):4-14.

46. Pignon JP, Bourhis J, Domenge C, Designé L. Chemotherapy added to locoregional treatment for head and neck squamous-cell carcinoma: three meta-analyses of updated individual data. $\mathrm{MACH}-\mathrm{NC}$ Collaborative Group. Meta-Analysis of Chemotherapy on Head and Neck Cancer. Lancet. 2000;355(9208):949-955.

47. Takagi M, Sakata K, Someya M, et al. Gimeracil sensitizes cells to radiation via inhibition of homologous recombination. Radiother Oncol. 2010;96(2):259-266.

48. Shirasaka T, Shimamoto Y, Fukushima M. Inhibition by oxonic acid of gastrointestinal toxicity of 5-fluorouracil without loss of its antitumor activity in rats. Cancer Res. 1993;53(17):4004-4009.

49. Ohashi T, Ohnishi M, Tanahashi S, Murai M. Efficacy and toxicity of concurrent chemoradiotherapy with nedaplatin and S-1 for head and neck cancer. Jpn J Clin Oncol. 2011;41(3):348-352.

50. Loehrer PJ Sr, Einhorn LH, Elson PJ, et al. A randomized comparison of cisplatin alone or in combination with methotrexate, vinblastine, and doxorubicin in patients with metastatic urothelial carcinoma: a cooperative group study. J Clin Oncol. 1992;10(7): 1066-1073.

51. von der Maase H, Sengelov L, Roberts JT, et al. Long-term survival results of a randomized trial comparing gemcitabine plus cisplatin, with methotrexate, vinblastine, doxorubicin, plus cisplatin in patients with bladder cancer. J Clin Oncol. 2005;23(21):4602-4608.

52. Pagliaro LC, Millikan RE, Tu SM, et al. Cisplatin, gemcitabine, and ifosfamide as weekly therapy: a feasibility and phase II study of salvage treatment for advanced transitional-cell carcinoma. J Clin Oncol. 2002; 20(13):2965-2970.

53. Shinohara N, Harabayashi T, Suzuki S, et al. Salvage chemotherapy with paclitaxel, ifosfamide, and nedaplatin in patients with urothelial cancer who had received prior cisplatin-based therapy. Cancer Chemother Pharmacol. 2006;58(3):402-407.

54. Furukawa T, Kubota T, Hoffman RM. Clinical applications of the histoculture drug response assay. Clin Cancer Res. 1995;1(3): 305-311.

55. Kitamura H, Taguchi K, Kunishima Y, et al. Paclitaxel, ifosfamide, and nedaplatin as second-line treatment for patients with metastatic urothelial carcinoma: a phase II study of the SUOC group. Cancer Sci. 2011;102(6):1171-1175.

56. Kurita H, Yamamoto E, Nozaki S, et al. Multicenter phase 2 study of induction chemotherapy with docetaxel and nedaplatin for oral squamous cell carcinoma. Cancer Chemother Pharmacol. 2010;65(3): 503-508. 


\section{Publish your work in this journal}

Cancer Management and Research is an international, peer-reviewed open access journal focusing on cancer research and the optimal use of preventative and integrated treatment interventions to achieve improved outcomes, enhanced survival and quality of life for the cancer patient. The journal welcomes original research, clinical \& epidemiological

studies, reviews \& evaluations, guidelines, expert opinion \& commentary, case reports \& extended reports. The manuscript management system is completely online and includes a very quick and fair peerreview system, which is all easy to use. Visit http://www.dovepress.com/ testimonials.php to read real quotes from published authors.

Submit your manuscript here: http://www.dovepress.com/cancer-management-and-research-journal 